Article

\title{
Generalized Neutrosophic Extended Triplet Group
}

\author{
Yingcang Ma ${ }^{1, *}$, Xiaohong Zhang ${ }^{2}$, Xiaofei Yang ${ }^{1}$ and Xin Zhou ${ }^{1}$ \\ 1 School of Science, Xi'an Polytechnic University, Xi'an 710048, China; \\ yangxiaofei2002@163.com (X.Y.); sxxzx1986@163.com (X.Z.) \\ 2 School of Arts and Sciences, Shaanxi University of Science \& Technology, Xi'an 710021, China; \\ zhangxiaohong@sust.edu.cn \\ * Correspondence: mayingcang@126.com
}

Received: 2 February 2019; Accepted: 25 February 2019; Published: 5 March 2019

\begin{abstract}
Neutrosophic extended triplet group is a new algebra structure and is different from the classical group. In this paper, the notion of generalized neutrosophic extended triplet group is proposed and some properties are discussed. In particular, the following conclusions are strictly proved: (1) an algebraic system is a generalized neutrosophic extended triplet group if and only if it is a quasi-completely regular semigroup; (2) an algebraic system is a weak commutative generalized neutrosophic extended triplet group if and only if it is a quasi-Clifford semigroup; (3) for each $n \in Z^{+}, n \geq 2,\left(Z_{n}, \otimes\right)$ is a commutative generalized neutrosophic extended triplet group; (4) for each $n \in Z^{+}, n \geq 2,\left(Z_{n}, \otimes\right)$ is a commutative neutrosophic extended triplet group if and only if $n=p_{1} p_{2} \cdots p_{m}$, i.e., the factorization of $n$ has only single factor.
\end{abstract}

Keywords: neutrosophic extended triplet group; generalized neutrosophic extended triplet group; quasi-completely regular semigroup; clifford semigroup

\section{Introduction}

Groups are very important algebraic structures and have been applied in many fields, such as cryptology, engineering, physics, chemistry, etc. Recently, a new algebraic system, neutrosophic triplet group (NTG), is proposed by Smarandache and Ali in [1]. For an NTG $(N, *)$, every element $a \in N$ has its own neutral element (denote by neut $(a)$ ) satisfying condition $a * n e u t(a)=\operatorname{neut}(a) * a=a$, and there exits at least one opposite element (denote by anti(a)) in $N$ relative to neut $(a)$ such that $a * \operatorname{anti}(a)=\operatorname{anti}(a) * a=\operatorname{neut}(a)$. Some further studies can be found in [2-11].

From the original definition of NTG, we can see that it is an extension of a group. However, it is different from a group. In NTG, the neutral element is different from the unit element of the classical algebraic system. By removing this restriction, an algebraic system, which is called neutrosophic extended triplet group (NETG), is proposed, and the classical group is regarded as a special case of NETG. Moreover, further studies of NETG can be found in [12-18] and some important results have been achieved.

For the algebraic system $\left(Z_{n}, \otimes\right), \otimes$ is the classical mod multiplication, where $Z_{n}=$ $\{[0],[1], \cdots,[n-1]\}$ and $n \geq 2$ is a positive integer. $\left(Z_{n}, \otimes\right)$ is only a commutative semigroup before NTG and NETG are introduced. Recently, properties of algebraic structure of $\left(Z_{n}, \otimes\right)$ in some special cases are studied in $[19,20]$ by the point view of NTG. We can see that for some positive integers $n,\left(Z_{n}, \otimes\right)$ is a commutative NETG, but for some positive integers $n,\left(Z_{n}, \otimes\right)$ is not a commutative NETG. So, there are two problems, 1 What conditions can guarantee $\left(Z_{n}, \otimes\right)$ to be a NETG? 2 Is there a new algebraic system that makes $\left(Z_{n}, \otimes\right)$ is the algebraic system for each positive integer $n$ ? We will give positive answers for the two problems in this paper.

The paper is organized as follows. Section 2 gives the related work. In Section 3, the conditions which guarantee the algebraic system $\left(Z_{n}, \otimes\right)$ to be a NETG are deeply studied. In Section 4 , 
the generalized neutrosophic extended triplet group (GNETG) is proposed and some properties and structure are discussed. Finally, the summary and future work are presented in Section 5.

\section{Related Works}

In this section, we will give the related research and results of the NETG. Some related notions are introduced at first. Let $G$ be non-empty set, $*$ is a binary operation on $G$. If $\forall a, b \in G$, implies $a * b \in G$, then $(G, *)$ is called a groupoid. A groupoid $G$ is called a semigroup if $*$ satisfies associative law, i.e., $(a * b) * c=a *(b * c)(\forall a, b, c \in G)$. A semigroup $G$ is called a group if there exists the unit element and for each element in $G$ exists its inverse element.

Definition 1. [11] Let $N$ be a non-empty set together with a binary operation $*$. Then, $N$ is called a neutrosophic extended triplet set if for any $a \in N$, there exists a neutral of " $a$ " (denote by neut $(a)$ ), and an opposite of " $a$ "(denote by anti $(a))$, such that neut $(a) \in N$, anti $(a) \in N$ and:

$$
a * \operatorname{neut}(a)=\operatorname{neut}(a) * a=a, a * \operatorname{anti}(a)=\operatorname{anti}(a) * a=\operatorname{neut}(a) .
$$

The triplet $(a$, neut $(a)$, anti $(a))$ is called a neutrosophic extended triplet.

Definition 2. [11,15] Let $(N, *)$ be a neutrosophic extended triplet set. Then, $N$ is called a neutrosophic extended triplet group (NETG), if the following conditions are satisfied:

(1) $(N, *)$ is well-defined, i.e., for any $a, b \in N$, one has $a * b \in N$.

(2) $(N, *)$ is associative, i.e., $(a * b) * c=a *(b * c)$ for all $a, b, c \in N$.

A NETG $N$ is called a commutative NETG if for all $a, b \in N, a * b=b * a$.

From Definition 2, we can see that a NETG is an extension of the classical group. There are some important results about the properties and structure of a NETG. For examples, $G$ is a NETG iff it is a completely regular semigroup, $G$ is a singular NETG iff it is a generalized group, and so on. We introduce some related notions and results in the following. Figure 1 gives the relations of the NETG and other algebraic structures.

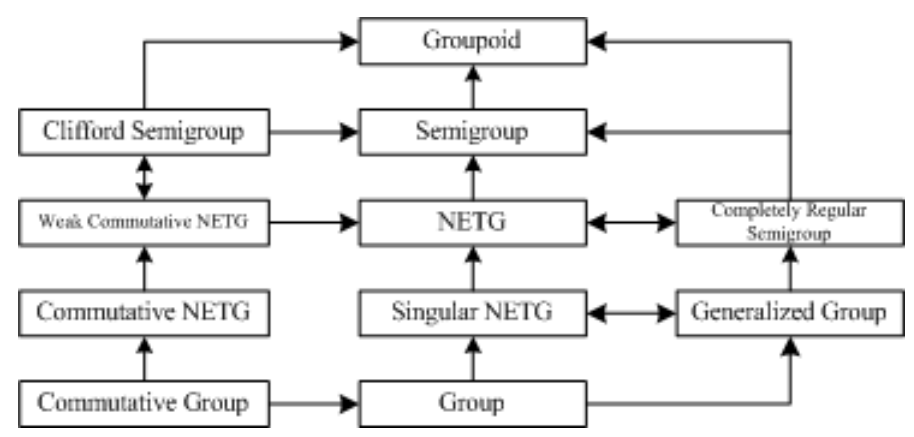

Figure 1. The relations of NETG and other algebraic structures.

Proposition 1. [15] $(N, *)$ be a NETG. We have:

(1) neut (a) is unique for any $a \in N$.

(2) neut $(a) *$ neut $(a)=$ neut $(a)$ for any $a \in N$.

(3) neut $($ neut $(a))=\operatorname{neut}(a)$ for any $a \in N$.

Definition 3. [17] A NETG $(N, *)$ is said to be singular, if anti(a) is unique for any $a \in N$.

Definition 4. [21] A generalized group $(G, *)$ is a non-empty set admitting a binary operation $*$ called multiplication subject to the set of rules given below:

(1) $(a * b) * c=a *(b * c), \forall a, b, c \in G$. 
(2) For each $a \in G$, there exists a unique $e(a) \in G$ such that $a * e(a)=e(a) * a=a$.

(3) For each $a \in G$, there exists $a^{-1} \in G$ such that $a * a^{-1}=a^{-1} * a=e(a)$.

Theorem 1. [17] $(N, *)$ is a singular NETG iff $(N, *)$ is a generalized group.

Definition 5. [15] Let $(N, *)$ be a NETG. Then $N$ is called a weak commutative NETG if a $*$ neut $(b)=$ neut $(b) * a$ for all $a, b \in N$.

Definition 6. [22] A semigroup $(S, *)$ is called regular if for any $a \in S$, there exists a unary operation $a \rightarrow a^{-1}$ on $S$ such that

$$
\left(a^{-1}\right)^{-1}=a, a * a^{-1} * a=a .
$$

A semigroup $S$ is called quasi-regular if for any $a \in S$, there exists a positive integer $n$ such that $a^{n}$ is regular.

Definition 7. [22] A semigroup $(S, *)$ is called completely regular if for any $a \in S$, there exists a unary operation $a \rightarrow a^{-1}$ on $S$ such that

$$
\left(a^{-1}\right)^{-1}=a, a * a^{-1} * a=a, a * a^{-1}=a^{-1} * a .
$$

A semigroup $S$ is called quasi-completely regular if for any $a \in S$, there exists a positive integer $n$ such that $a^{n}$ is completely regular.

Proposition 2. [22] Let $(S, *)$ be a semigroup. Then the following statements are equivalent:

(1) $S$ is completely regular;

(2) every element of $S$ lies in a subgroup of $S$;

(3) every $H$-class in $S$ is a group.

Definition 8. [22] A semigroup $(S, *)$ is called Clifford semigroup, if it is completely regular and in which for any $a, b \in S$, such that

$$
\left(a * a^{-1}\right) *\left(b * b^{-1}\right)=\left(b * b^{-1}\right) *\left(a * a^{-1}\right) .
$$

Proposition 3. [22] Let $(S, *)$ be a semigroup. Then the following statements are equivalent:

(1) $S$ is Clifford semigroup;

(2) $S$ is a semilattice of groups;

(3) $S$ is regular, and the idempotents of $S$ are central.

Theorem 2. [18] Let $(N, *)$ be a groupoid. Then $N$ is a NETG iff it is a completely regular semigroup.

Theorem 3. [18] Let $(N, *)$ be a groupoid. Then $N$ is a weak commutative NETG iff it is a Clifford semigroup.

3. The Relations of $\left(Z_{n}, \otimes\right)$ and NETG

Lemma 1. In an algebra system $\left(Z_{n}, \otimes\right)$, where $Z$ is the integer set, we have for $a, b, c \in Z, a b \equiv c(\bmod n)$ holds iff $[a] \otimes[b]=[c]$ holds.

Lemma 2. For each positive integer $n \geq 2$, linear congruence equation $a x \equiv c(\bmod n)$ has a solution iff $\operatorname{gcd}(a, n) \mid c$, where $\operatorname{gcd}(a, b)$ is the greatest common divisor of $a$ and $b$.

Proposition 4. The solution of $x$ for equation $[a] \otimes[x]=[a]$ is $x=m n / \operatorname{gcd}(a, n)+1$, where $m \in Z$.

Proof. From Lemma $1,[a] \otimes[x]=[a]$ has a solution iff $a x \equiv a(\bmod n)$ has a solution, and by Lemma 2, $a x \equiv a(\bmod n)$ must have a solution being $\operatorname{gcd}(a, n) \mid a$ holds obviously. We will prove the solution to the equation $[a] \otimes[x]=[a]$ is $m n / \operatorname{gcd}(a, n)$ in the following. 
Let $\operatorname{gcd}(a, n)=d$, then there exist two integers $a_{1}$ and $n_{1}$, such that $a=a_{1} * d, n=n_{1} * d$ and $\operatorname{gcd}\left(a_{1}, n_{1}\right)=1$. If $x$ satisfies the equation $a x \equiv a(\bmod n)$, we can get that $n \mid(a x-a)$, thus $n_{1} \mid\left(a_{1} x-a_{1}\right)$, i.e., $a_{1} x-a_{1}=m_{1} n_{1}, m_{1} \in Z$, that is $a_{1}(x-1)=m_{1} n_{1}$. Since $\operatorname{gcd}\left(a_{1}, n_{1}\right)=1$, we have $a_{1} \mid m_{1}$. Let $m_{1}=m a_{1}, m \in Z$, then, $a_{1}(x-1)=m a_{1} n_{1}$, that is $x=m n_{1}+1, m \in Z$.

On the other hand, being $a\left(m n_{1}+1\right)-a=a m n_{1}=a_{1} m n$, that is $n \mid\left(a\left(m n_{1}+1\right)-a\right)$, i.e., $a\left(m n_{1}+\right.$ $1) \equiv a(\bmod n)$ holds, so $x=m n_{1}+1$ is the solution of equation $a x \equiv a(\bmod n)$. This tells us that the solution for equation $[a] \otimes[x]=[a]$ is $x=m n / \operatorname{gcd}(a, n)+1$, where $m \in Z$.

Remark: From Proposition 4, we can see that the solution of $[a] \otimes[x]=[a]$ is just decided by the $\operatorname{gcd}(a, n)$. If $\operatorname{gcd}(a, n)=\operatorname{gcd}(b, n)$, then $[a] \otimes[x]=[a]$ and $[b] \otimes[x]=[b]$ have the same solution in $\left(Z_{n}, \otimes\right)$.

Theorem 4. In the algebra system $\left(Z_{n}, \otimes\right)$, for each $[a] \in Z_{n},[a]$ has neut $([a])$ and anti $([a])$ iff $\operatorname{gcd}(\operatorname{gcd}(a, n), n / \operatorname{gcd}(a, n))=1$.

Proof. Necessity: In general, let $[a] \in Z_{n}$ and the neutral elements of $[a]$ exists, that is there are neut $([a])$ and anti $([a])$ such that $[a] \otimes \operatorname{neut}([a])=[a]$ and $[a] \otimes \operatorname{anti}([a])=\operatorname{neut}([a])$. That is the linear congruence equations $a x \equiv a(\bmod n)$ and $a y \equiv x(\bmod n)$ have solutions. Let $\operatorname{gcd}(a, n)=d$ and $a=a_{1} * d, n=n_{1} * d$. From Proposition 4, $x=m n_{1}+1, m \in Z$. From Lemma 2, $y$ has solution for equation $a y \equiv x(\bmod n)$ iff $\operatorname{gcd}(a, n) \mid x$, that is $\operatorname{gcd}(a, n) \mid\left(m n_{1}+1\right)$, which means $\operatorname{gcd}(\operatorname{gcd}(a, n), n / \operatorname{gcd}(a, n))=1$.

Sufficiency: If $\operatorname{gcd}(\operatorname{gcd}(a, n), n / \operatorname{gcd}(a, n))=1$, we should verify that there is $x$ and $y$, such that $a x \equiv a(\bmod n)$ and $a y \equiv x(\bmod n)$. From Proposition $4, a x \equiv a(\bmod n)$ has a solution and $x=$ $m n_{1}+1, m \in Z$. From the given condition $\operatorname{gcd}\left(d, n_{1}\right)=1$, we can infer that there exist $p, q \in Z$, such that $p d+q n_{1}=1$, which means $d \mid\left((-q) n_{1}+1\right)$, that is $d \mid x$ when $m=-q$. So there exists $y$, such that ay $\equiv x(\bmod n)$, which means if $\operatorname{gcd}(\operatorname{gcd}(a, n), n / \operatorname{gcd}(a, n))=1$, thus for $[a] \in Z_{n}$, [a] has neut $([a])$ and $\operatorname{anti}([a])$.

Example 1. For $\left(Z_{8}, \otimes\right), n=8=2^{3}$, the operation table as following Table 1. From Definition 1 , we have netu $([0])=[0],\{\operatorname{anti}([0])\}=\{[0],[1],[2],[3],[4],[5],[6],[7]\}, \operatorname{netu}([1])=[1]$, anti $([1])=$ $[1], \operatorname{netu}([3])=[1], \operatorname{anti}([3])=[3], \operatorname{netu}([5])=[1], \operatorname{anti}([5])=[5], \operatorname{netu}([7])=[1], \operatorname{anti}([7])=[7]$, but [2], [4] and [6] have not the neutral element and opposite element.

We can get the above results by Theorem 4. Being $\operatorname{gcd}(\operatorname{gcd}(1,8), 8 / \operatorname{gcd}(1,8))=$ $\operatorname{gcd}(\operatorname{gcd}(3,8), 8 / \operatorname{gcd}(3,8))=\operatorname{gcd}(\operatorname{gcd}(5,8), 8 / \operatorname{gcd}(5,8))=\operatorname{gcd}(\operatorname{gcd}(7,8), 8 / \operatorname{gcd}(7,8))=1$, so from Theorem 4, [1], [3], [5] and [7] exist the neutral element and opposite element. In fact, from Proposition 4, being $\operatorname{gcd}(1,8)=\operatorname{gcd}(3,8)=\operatorname{gcd}(5,8)=\operatorname{gcd}(7,8)=1$, i.e., they have the same greatest common divisor, so they have the same neutral element, that is [1]. In the same way, [0] has its neutral element and opposite element. However, $\operatorname{gcd}(\operatorname{gcd}(2,8), 8 / \operatorname{gcd}(2,8))=\operatorname{gcd}(\operatorname{gcd}(4,8), 8 / \operatorname{gcd}(4,8))=\operatorname{gcd}(\operatorname{gcd}(6,8), 8 / \operatorname{gcd}(6,8))=$ $2 \neq 1$, so [2], [4] and [6] do not have the neutral element and opposite element.

Table 1. The operation table of $Z_{8}$.

\begin{tabular}{ccccccccc}
\hline$\otimes$ & {$[0]$} & {$[1]$} & {$[2]$} & {$[3]$} & {$[4]$} & {$[5]$} & {$[6]$} & {$[7]$} \\
\hline$[0]$ & {$[0]$} & {$[0]$} & {$[0]$} & {$[0]$} & {$[0]$} & {$[0]$} & {$[0]$} & {$[0]$} \\
{$[1]$} & {$[0]$} & {$[1]$} & {$[2]$} & {$[3]$} & {$[4]$} & {$[5]$} & {$[6]$} & {$[7]$} \\
{$[2]$} & {$[0]$} & {$[2]$} & {$[4]$} & {$[6]$} & {$[0]$} & {$[2]$} & {$[4]$} & {$[6]$} \\
{$[3]$} & {$[0]$} & {$[3]$} & {$[6]$} & {$[1]$} & {$[4]$} & {$[7]$} & {$[2]$} & {$[5]$} \\
{$[4]$} & {$[0]$} & {$[4]$} & {$[0]$} & {$[4]$} & {$[0]$} & {$[4]$} & {$[0]$} & {$[4]$} \\
{$[5]$} & {$[0]$} & {$[5]$} & {$[2]$} & {$[7]$} & {$[4]$} & {$[1]$} & {$[6]$} & {$[3]$} \\
{$[6]$} & {$[0]$} & {$[6]$} & {$[4]$} & {$[2]$} & {$[0]$} & {$[6]$} & {$[4]$} & {$[2]$} \\
{$[7]$} & {$[0]$} & {$[7]$} & {$[6]$} & {$[5]$} & {$[4]$} & {$[3]$} & {$[2]$} & {$[1]$} \\
\hline
\end{tabular}


Proposition 5. In the algebra system $\left(Z_{n}, \otimes\right)$, if the neutral element exists, then it is unique.

Proof. In general, if $[a] \in Z_{n}$ and the neutral elements of $[a]$ exists, that is there are neut $([a])$ and $\operatorname{anti}([a])$ such that $[a] \otimes$ neut $([a])=[a]$ and $[a] \otimes \operatorname{anti}([a])=\operatorname{neut}([a])$. Let $\operatorname{gcd}(a, n)=d$ and there are two integers $a_{1}$ and $n_{1}$, such that $a=a_{1} * d, n=n_{1} * d$.

By Proposition 4, the neutral element of $[a]$ has form $n_{1} m+1$, where $m \in Z$, and from Theorem 4 , there exists $m_{0} \in Z$ such that $d \mid\left(n_{1} m_{0}+1\right)$. Since $d \mid n$, we have $d \mid\left(n_{1} m_{0}+1 \pm n\right)$, which means there must exist $m \in Z$ such that $0 \leq n_{1} m+1<n$, so the neutral element of $[a]$ exists.

In the following, we will show that if there exist two integers $m_{1}$ and $m_{2}$, such that $d \mid\left(n_{1} m_{1}+1\right)$ and $d \mid\left(n_{1} m_{2}+1\right)$, then $n \mid n_{1}\left(m_{1}-m_{2}\right)$.

From $d \mid\left(n_{1} m_{1}+1\right)$ and $d \mid\left(n_{1} m_{2}+1\right)$, we have $d \mid\left(n_{1} m_{1}+1-n_{1} m_{2}-1\right)$, i.e., $d \mid\left(n_{1}\left(m_{1}-m_{2}\right)\right)$. Being $\operatorname{gcd}\left(d, n_{1}\right)=1$, then $d \mid\left(m_{1}-m_{2}\right)$, that is $n \mid n_{1}\left(m_{1}-m_{2}\right)$.

From the above analysis, we can get that the neutral element of $[a]$ is unique in $\left(Z_{n}, \otimes\right)$ if it exists.

Remark: From Proposition 5, we can see that if the neutral element of $[a]$ exists, then it is unique. Let neut $([a])$ is the neutral element of $[a]$, then we have that (neut $([a]$, neut $([a])),[1])$ and $($ neut $([a]$, neut $([a]))$, neut $([a]))$ are the neutrosophic extended triplets in $\left(Z_{n}, \otimes\right)$.

Example 2. For $\left(Z_{36}, \otimes\right), n=36=2^{2} 3^{2}$. From the above results we have:

(1) [2] has not the neutral element and opposite element being $\operatorname{gcd}(\operatorname{gcd}(2,36), 36 / \operatorname{gcd}(2,36))=2 \neq 1$. In fact, [2], [3], [6], [10], [12], [14], [15], [18], [21], [22], [24], [26], [30], [33] and [34] do not have the neutral element and opposite element by Theorem 4 for the same reason.

(2) [4] and [8] have the same neutral element being $\operatorname{gcd}(4,36)=\operatorname{gcd}(8,36)=4$. In fact, [4], [8], [16], [20], [28] and [32] have the same neutral element, which is [28].

(3) [9] and [27] have the same neutral element [9] being $\operatorname{gcd}(9,36)=\operatorname{gcd}(27,36)=9$.

(4) [1], [5], [7], [11], [13], [17], [19], [23], [25], [29], [31] and [35] have the same neutral element [1].

(5) [0] has the neutral element [0].

(6) We can see that the neutral element of each element is unique and explain that Proposition 5 is correct. Moreover, $([0],[0],[1]),([0],[0],[0]),([1],[1],[1]),([9],[9],[1]),([9],[9],[9]),([28],[28],[1])$ and $([28],[28],[28])$ are some neutrosophic extended triplets in $\left(Z_{36}, \otimes\right)$, which verify the above Remark.

Theorem 5. An algebra system $\left(Z_{n}, \otimes\right)$ is a NETG iff the factorization of $n$ is a product of single factors, i.e., $n=p_{1} p_{2} \cdots p_{k}$, where $p_{i}(i=1,2, \cdots, k)$ is a prime number.

Proof. Necessity: Suppose that $\left(Z_{n}, \otimes\right)$ is a NETG, we verify the factorization of $n$ is the product of a single factor and proof by contradiction.

Assume, the factorization of $n$ is $n=p_{1}^{k_{1}} p_{2}^{k_{2}} \cdots p_{t}^{k_{t}}$, and $\exists i$, such that $k_{i} \geq 2$. Without losing generality, let $k_{1} \geq 2$. We will prove neut $\left(\left[p_{1}\right]\right)$ and anti $\left(\left[p_{1}\right]\right)$ do not exist.

Because $\operatorname{gcd}\left(\operatorname{gcd}\left(p_{1}, n\right), n / \operatorname{gcd}\left(p_{1}, n\right)\right)=p_{1}^{k_{1}-1}$ and $k_{1} \geq 2$, then

$$
\operatorname{gcd}\left(\operatorname{gcd}\left(p_{1}, n\right), n / \operatorname{gcd}\left(p_{1}, n\right)\right) \neq 1
$$

By Theorem 4, we have that neut $\left(\left[p_{1}\right]\right)$ and anti $\left(\left[p_{1}\right]\right)$ do not exist. This contradicts that $\left(Z_{n}, \otimes\right)$ is NETG. So, the factorization of $n$ is a product of single factors if $\left(Z_{n}, \otimes\right)$ is a NETG.

Sufficiency: If the factorization of $n$ is a product of some single factors, i.e., $n=p_{1} p_{2} \cdots p_{k}$, where $p_{i}(i=1,2, \cdots, k)$ is a prime number. We will prove for each $[a] \in Z_{n}$, neut $([a])$ and $\operatorname{anti}([a])$ exist.

Let $d=\operatorname{gcd}(a, n)$, because $n=p_{1} p_{2} \cdots p_{k}, \operatorname{sog} \operatorname{gcd}(d, n / d)=1$, i.e.,

$$
\operatorname{gcd}(\operatorname{gcd}(a, n), n / \operatorname{gcd}(a, n))=1 \text {. }
$$


From Theorem 4, $[a]$ has the neutral element and opposite element. From the arbitrariness of $a$, we can know that $\left(Z_{n}, \otimes\right)$ is a NETG if the factorization of $n$ is a product of some single factors.

Remark: For an algebra system $\left(Z_{n}, \otimes\right)$ and $n=p_{1} p_{2} \cdots p_{k}$, where $p_{i}(i=1,2, \cdots, k)$ is a prime number. From the above analysis, the number of different neutral elements in $Z_{n}$ is $2^{k}$.

Example 3. For $\left(Z_{30}, \otimes\right), n=30=2^{1} 3^{1} 5^{1}$, so each element in $Z_{30}$ has its corresponding neutral element. From the above Remark, the number of different neutral elements in $Z_{30}$ is $2^{3}=8$. In fact, being different factors of 30 are $1,2,3,5,6,10,15$, and 30 . Thus, the different neutral elements are [1], [16], [21], [25], [6], [10], [15] and $[0]$ respectively. In detail (just consider the neutral element):

(1) [1], [7], [11], [13], [17], [19], [23] and [29] have the same neutral element, which is [1].

(2) $[2],[4],[8],[14],[16],[22],[26]$ and [28] have the same neutral element, which is [16].

(3) [3], [9], [21] and [27] have the same neutral element, which is [21].

(4) [5] and [25] have the same neutral element, which is [25].

(5) [6], [12], [18] and [24] have the same neutral element, which is [6].

(6) [10] and [20] have the same neutral element, which is [10].

(7) [15] has neutral element [15].

(8) [0] has neutral element [0].

\section{GNETG and Quasi-Completely Regular Semigroup}

Definition 9. Let $N$ be a non-empty set together with a binary operation $*$. Then, $N$ is called a generalized neutrosophic extended triplet set if for any $a \in N$, there exist at least a positive integer $n$, such that $a^{n}$ exists neutral element, denoted by neut $\left(a^{n}\right)$, and opposite element, denoted by anti $\left(a^{n}\right)$. The triplet $\left(a\right.$, neut $\left(a^{n}\right)$, anti $\left.\left(a^{n}\right)\right)$ is called a generalized neutrosophic extended triplet with degree $n$.

Definition 10. Let $(N, *)$ is a generalized neutrosophic extended triplet set. Then, $N$ is called a GNETG, if the following conditions are satisfied:

(1) $(N, *)$ is well-defined, i.e., for any $a, b \in N, a * b \in N$.

(2) $(N, *)$ is associative, i.e., $(a * b) * c=a *(b * c)$ for all $a, b, c \in N$.

A GNETG $N$ is called a commutative generalized neutrosophic extended triplet group if for all $a, b \in$ $N, a * b=b * a$.

Remark: From Definition 9, it is obviously that a neutrosophic extended triplet is a generalized neutrosophic extended triplet with degree 1, so a neutrosophic extended triplet set is a generalized neutrosophic extended triplet set. Moreover, a NETG is a GNETG, but a GNETG is not a NETG in general.

Example 4. Let $S=\{a, e, f, g\}$, an operation $*$ on $S$ is defined as in Table 2. We can see that $(e, e, a),(e, e, e),(f, f, f)$ and $(g, g, g)$ are neutrosophic extended triplets, but a does not exist the neutral element and opposite element. Thus, $S$ is not a NETG. Moreover, $a^{2}=e$ has the neutral element and opposite element, so $(S, *)$ is a GNETG. $(a, e, a)$ and $(a, e, e)$ are generalized neutrosophic extended triplets with degree 2. We can infer that $(S, *)$ is a GNETG but not a NETG. Moreover, it is not a commutative GNETG being $e * f \neq f * e$.

Table 2. A GNETG of Example 4.

\begin{tabular}{lllll}
\hline$*$ & $a$ & $e$ & $f$ & $g$ \\
\hline$a$ & $e$ & $e$ & $g$ & $e$ \\
$e$ & $e$ & $e$ & $e$ & $e$ \\
$f$ & $f$ & $f$ & $f$ & $f$ \\
$g$ & $g$ & $g$ & $g$ & $g$ \\
\hline
\end{tabular}


Example 5. Consider $\left(Z_{12}, \otimes\right), 12=2^{2} 3^{1}$. From Theorem 4, we have:

(1) $[0],[1],[3],[4],[5],[7],[8],[9]$ and [11] exist the neutral element and opposite element.

(2) [2] does not exist the neutral element and opposite element, but we can see that $[2]^{2}=[4]$ exists the neutral element and opposite element.

(3) [6] does not exist the neutral element and opposite element, but we can see that $[6]^{2}=[0]$ exists the neutral element and opposite element.

(4) [10] does not exist the neutral element and opposite element, but we can see that $[10]^{2}=[4]$ exists the neutral element and opposite element.

So $Z_{12}$ is a generalized neutrosophic extended triplet set, but it is not a neutrosophic extended triplet set. Moreover, $\left(Z_{12}, \otimes\right)$ is a commutative GNETG. The following theorem shows that for each positive integer $n \geq 2,\left(Z_{n}, \otimes\right)$ is a commutative GNETG.

Theorem 6. For each $[a] \in Z_{n}$, there exists a positive integer $m$, such that $[a]^{m}$ has the neutral element and opposite element. That is, $\left(Z_{n}, \otimes\right)$ is commutative GNETG.

Proof. If the factorization of $n$ is a product of single factors, From Theorem $5,\left(Z_{n}, \otimes\right)$ is a NETG, so $[a]^{1}=[a]$ has the neutral element and opposite element.

If $n=p_{1}^{k_{1}} p_{2}^{k_{2}} \cdots p_{t}^{k_{t}}$, and $\exists i$, such that $k_{i} \geq 2$. For each $[a] \in Z_{n}$, it is easy to get that there is a positive integer $m$, such that

$$
\operatorname{gcd}\left(\left(\operatorname{gcd}\left(a^{m}, n\right)\right), n /\left(\operatorname{gcd}\left(a^{m}, n\right)\right)\right)=1,
$$

that is $\left[a^{m}\right]=[a]^{m}$ has the neutral element and opposite element from Theorem 4 . So, for each $[a] \in Z_{n}$, there exists a positive integer $m$, such that $[a]^{m}$ has the neutral element and opposite element, i.e., $\left(Z_{n}, \otimes\right)$ is a commutative GNETG.

Definition 11. [22] Let $(S, *)$ be a semigroup, an element $x$ in $S$ is said to be periodic if there exists a positive integer $n$ such that $x^{n+1}=x$. S is pointwise periodic if each $x$ in $S$ is periodic.

Proposition 6. Let $(S, *)$ be a pointwise periodic semigroup, then $(S, *)$ is a NETG, so it is a GNETG.

Proof. Since $(S, *)$ is a pointwise periodic semigroup, so for each element $x$ in a $S$, there exists a positive integer $n$ such that $x^{n+1}=x$.

If $n=1$, then $x^{2}=x$, so $(x, x, x)$ is a neutrosophic extended triplet.

If $n \geq 2$, so $x * x^{n}=x^{n} * x=x$ and $x * x^{n-1}=x^{n-1} * x=x^{n}$, that is, $\left(x, x^{n}, x^{n-1}\right)$ is a neutrosophic extended triplet.

By the arbitrariness of $x$, we have $(S, *)$ is a NETG. Of course, it is a GNETG.

Example 6. Let $S=\{a, b, c\}$, an operation $*$ on $S$ is defined as following Table 3. Because $a^{2}=a, b^{3}=$ $b, c^{2}=c$, so $(S, *)$ is a pointwise periodic semigroup. Moreover, $(a, a, a),(b, a, b),(c, c, a),(c, c, b)$ and $(c, c, c)$ are neutrosophic extended triplets, that is $(S, *)$ is a NETG.

Table 3. A pointwise periodic semigroup of Example 6.

\begin{tabular}{llll}
\hline$*$ & $a$ & $b$ & $c$ \\
\hline$a$ & $a$ & $b$ & $c$ \\
$b$ & $b$ & $a$ & $c$ \\
$c$ & $c$ & $c$ & $c$ \\
\hline
\end{tabular}

From Example 6, we can also see that if $(S, *)$ has zero element ( $c$ is the zero element in Example 6), then the neutral element of zero element is itself and the opposite element of zero element is every element in $S$. 
Proposition 7. Let $(N, *)$ is a GNETG, $a \in N$ and $\left(a\right.$, neut $\left(a^{n}\right)$, anti $\left.\left(a^{n}\right)\right)$ is a generalized neutrosophic extended triplet with degree $n$. We have:

(1) neut $\left(a^{n}\right)$ is unique.

(2) neut $\left(a^{n}\right) * \operatorname{neut}\left(a^{n}\right)=\operatorname{neut}\left(a^{n}\right)$.

Proof. Assume $c, d \in\left\{\right.$ neut $\left.\left(a^{n}\right)\right\}$, so $a^{n} * c=c * a^{n}=a^{n}, a^{n} * d=d * a^{n}=a^{n}$, and there exists $x, y \in N$ such that

$$
a^{n} * x=x * a^{n}=c, a^{n} * y=y * a^{n}=d .
$$

We can obtain

$$
\begin{gathered}
c * d=\left(x * a^{n}\right) * d=x *\left(a^{n} * d\right)=x * a^{n}=c, \\
c * d=\left(a^{n} * x\right) *\left(a^{n} * y\right)=\left(a^{n} *\left(x * a^{n}\right)\right) * y=d .
\end{gathered}
$$

We have $c=d=c * d$. So neut $\left(a^{n}\right)$ is unique and neut $\left(a^{n}\right) * \operatorname{neut}\left(a^{n}\right)=\operatorname{neut}\left(a^{n}\right)$.

Proposition 8. Let $(N, *)$ is a GNETG, $a \in N$ and $\left(a\right.$, neut $\left(a^{n}\right)$, anti $\left.\left(a^{n}\right)\right)$ is a generalized neutrosophic extended triplet with degree $n$. Then

(1) $a^{n} * x * \operatorname{neut}\left(a^{n}\right)=x * \operatorname{neut}\left(a^{n}\right) * a^{n}=\operatorname{neut}\left(a^{n}\right)$, for any $x \in\left\{\operatorname{anti}\left(a^{n}\right)\right\}$.

(2) $a^{n} * \operatorname{neut}\left(a^{n}\right) * x=\operatorname{neut}\left(a^{n}\right) * x * a^{n}=\operatorname{neut}\left(a^{n}\right)$ for any $x \in\left\{\operatorname{anti}\left(a^{n}\right)\right\}$.

(3) neut $\left(\left(\operatorname{neut}\left(a^{n}\right)\right)\right)=\operatorname{neut}\left(x * \operatorname{neut}\left(a^{n}\right)\right)=\operatorname{neut}\left(a^{n}\right), x \in\left\{\operatorname{anti}\left(a^{n}\right)\right\} \cup\left\{a^{n}\right\}$.

(4) neut $\left(a^{n}\right) * x=y * \operatorname{neut}\left(a^{n}\right)=z *$ neut $\left(a^{n}\right)$, for any $x, y, z \in\left\{\operatorname{anti}\left(a^{n}\right)\right\}$.

(5) $\operatorname{anti}\left(x * \operatorname{neut}\left(a^{n}\right)\right) * \operatorname{neut}\left(x * \operatorname{neut}\left(a^{n}\right)\right)=a^{n}$, for any $x \in\left\{\operatorname{anti}\left(a^{n}\right)\right\}$.

Proof. (1) For any $x \in\left\{\operatorname{anti}\left(a^{n}\right)\right\}$, from Definition 9 and Proposition 7, we have

$$
\begin{gathered}
a^{n} * x * \operatorname{neut}\left(a^{n}\right)=\left(a^{n} * x\right) * \operatorname{neut}\left(a^{n}\right)=\operatorname{neut}\left(a^{n}\right) * \operatorname{neut}\left(a^{n}\right)=\operatorname{neut}\left(a^{n}\right), \\
\left.x * \operatorname{neut}\left(a^{n}\right) * a^{n}=x * \operatorname{neut}\left(a^{n}\right) * a^{n}\right)=x * a^{n}=\operatorname{neut}\left(a^{n}\right) .
\end{gathered}
$$

So $a^{n} * x * \operatorname{neut}\left(a^{n}\right)=x * \operatorname{neut}\left(a^{n}\right) * a^{n}=\operatorname{neut}\left(a^{n}\right)$, for any $x \in\left\{\operatorname{anti}\left(a^{n}\right)\right\}$.

(2) For any $x \in\left\{\operatorname{anti}\left(a^{n}\right)\right\}$, From Definition 9 and Proposition 7, we have

$$
\begin{gathered}
a^{n} * \operatorname{neut}\left(a^{n}\right) * x=\left(a^{n} * \operatorname{neut}\left(a^{n}\right)\right) * x=a^{n} * x=\operatorname{neut}\left(a^{n}\right), \\
\operatorname{neut}\left(a^{n}\right) * x * a^{n}=\operatorname{neut}\left(a^{n}\right) *\left(x * a^{n}\right)=\operatorname{neut}\left(a^{n}\right) * \operatorname{neut}\left(a^{n}\right)=\operatorname{neut}\left(a^{n}\right) .
\end{gathered}
$$

So $a^{n} * \operatorname{neut}\left(a^{n}\right) * x=\operatorname{neut}\left(a^{n}\right) * x * a^{n}=\operatorname{neut}\left(a^{n}\right)$ for any $x \in\left\{\operatorname{anti}\left(a^{n}\right)\right\}$.

(3) We prove neut $\left(a^{n}\right)=\operatorname{neut}\left(\left(\right.\right.$ neut $\left.\left.\left(a^{n}\right)\right)\right)$ firstly.

For any $x \in\left\{\operatorname{anti}\left(a^{n}\right)\right\}$ and $y \in\left\{\operatorname{anti}\left(\right.\right.$ neut $\left.\left.\left(a^{n}\right)\right)\right\}$,

$$
(y * x) * a^{n}=y *\left(x * a^{n}\right)=y * \operatorname{neut}\left(a^{n}\right)=\operatorname{neut}\left(\operatorname{neut}\left(a^{n}\right)\right) .
$$

Moreover,

$$
\left((y * x) * a^{n}\right) * \operatorname{neut}\left(a^{n}\right)=(y * x) *\left(a^{n} * \operatorname{neut}\left(a^{n}\right)\right)=(y * x) * a^{n}=\operatorname{neut}\left(\operatorname{neut}\left(a^{n}\right)\right) .
$$

Thus, neut $\left(a^{n}\right)=\operatorname{neut}\left(\operatorname{neut}\left(a^{n}\right)\right) * \operatorname{neut}\left(a^{n}\right)=\left((y * x) * a^{n}\right) * \operatorname{neut}\left(a^{n}\right)=\operatorname{neut}\left(\operatorname{neut}\left(a^{n}\right)\right)$.

In the following we will prove neut $\left(x *\right.$ neut $\left.\left(a^{n}\right)\right)=$ neut $\left(a^{n}\right), x \in\left\{\operatorname{anti}\left(a^{n}\right)\right\} \cup\left\{a^{n}\right\}$.

It is obvious that neut $\left(x * \operatorname{neut}\left(a^{n}\right)\right)=\operatorname{neut}\left(a^{n}\right)$ when $x=a^{n}$. If $x \in\left\{\operatorname{anti}\left(a^{n}\right)\right\}$, by Proposition 7 we have:

$$
\left(x * \operatorname{neut}\left(a^{n}\right)\right) * \operatorname{neut}\left(a^{n}\right)=x *\left(\operatorname{neut}\left(a^{n}\right) * \operatorname{neut}\left(a^{n}\right)\right)=x * \operatorname{neut}\left(a^{n}\right),
$$




$$
\begin{aligned}
\operatorname{neut}\left(a^{n}\right) *\left(x * \operatorname{neut}\left(a^{n}\right)\right) & =\left(\operatorname{neut}\left(a^{n}\right) * x\right) * \operatorname{neut}\left(a^{n}\right)=\left(x * \operatorname{neut}\left(a^{n}\right)\right) * \operatorname{neut}\left(a^{n}\right) \\
& =x *\left(\operatorname{neut}\left(a^{n}\right) * \operatorname{neut}\left(a^{n}\right)\right)=x * \operatorname{neut}\left(a^{n}\right) .
\end{aligned}
$$

Moreover, we can get

$$
\begin{gathered}
\left(x * \operatorname{neut}\left(a^{n}\right)\right) * a^{n}=x *\left(\operatorname{neut}\left(a^{n}\right) * a^{n}\right)=x * a^{n}=\operatorname{neut}\left(a^{n}\right), \\
a^{n} *\left(x * \operatorname{neut}\left(a^{n}\right)\right)=\left(a^{n} * x\right) * \operatorname{neut}\left(a^{n}\right)=\operatorname{neut}\left(a^{n}\right) * \operatorname{neut}\left(a^{n}\right)=\operatorname{neut}\left(a^{n}\right) .
\end{gathered}
$$

Thus, neut $\left(a^{n}\right)=\operatorname{neut}\left(x *\right.$ neut $\left.\left(a^{n}\right)\right)$.

(4) For any $x, y, z \in\left\{\operatorname{anti}\left(a^{n}\right)\right\}$, we have

$$
\operatorname{neut}\left(a^{n}\right) * x=\left(y * a^{n}\right) * x=y *\left(a^{n} * x\right)=y * \operatorname{neut}\left(a^{n}\right) .
$$

Moreover,

$$
y * \operatorname{neut}\left(a^{n}\right)=y *\left(a^{n} * z\right)=\left(y * a^{n}\right) * z=\operatorname{neut}\left(a^{n}\right) * z=\left(z * a^{n}\right) * z=z *\left(a^{n} * z\right)=z * \operatorname{neut}\left(a^{n}\right) .
$$

Thus, (4) holds.

(5) Suppose $x \in\left\{\operatorname{anti}\left(a^{n}\right)\right\}$, for each $y \in\left\{\operatorname{anti}\left(x *\right.\right.$ neut $\left.\left.\left(a^{n}\right)\right)\right\}$, from (4) we know that anti $(x *$ neut $\left.\left(a^{n}\right)\right) * \operatorname{neut}\left(x *\right.$ neut $\left.\left(a^{n}\right)\right)$ is unique. Applying $(1), a^{n} \in\left\{\operatorname{anti}\left(x *\right.\right.$ neut $\left.\left.\left(a^{n}\right)\right)\right\}$, that is,

$$
\operatorname{anti}\left(x * \operatorname{neut}\left(a^{n}\right)\right) * \operatorname{neut}\left(x * \operatorname{neut}\left(a^{n}\right)\right)=a^{n} \text { neut }\left(x * \operatorname{neut}\left(a^{n}\right)\right) .
$$

By (3), neut $\left(x * \operatorname{neut}\left(a^{n}\right)\right)=\operatorname{neut}\left(a^{n}\right)$. Thus, anti $\left(x * \operatorname{neut}\left(a^{n}\right)\right) * \operatorname{neut}\left(x * \operatorname{neut}\left(a^{n}\right)\right)=a^{n} * \operatorname{neut}(x *$ $\left.\operatorname{neut}\left(a^{n}\right)\right)=a^{n} * \operatorname{neut}\left(a^{n}\right)=a^{n}$.

Example 7. Let $S=\{a, e, b, f, c, g\}$, an operation $*$ on $S$ is defined as following Table 4. Since neut $(e)=$ $e,\{\operatorname{anti}(e)\}=\{a, e\}, \operatorname{neut}(f)=f,\{\operatorname{anti}(f)\}=\{b, f\}, \operatorname{neut}(g)=g,\{\operatorname{anti}(g)\}=\{a, e, b, f, c, g\}$ and $a^{2}=e, b^{2}=f, c^{2}=g$, so $(S, *)$ is a GNETG. We can get that (Corresponding to the results of Proposition 8):

Table 4. A GNETG of Example 7.

\begin{tabular}{lllllll}
\hline$*$ & $a$ & $e$ & $b$ & $f$ & $c$ & $g$ \\
\hline$a$ & $e$ & $e$ & $c$ & $c$ & $c$ & $g$ \\
$e$ & $e$ & $e$ & $c$ & $c$ & $c$ & $g$ \\
$b$ & $g$ & $g$ & $f$ & $f$ & $g$ & $g$ \\
$f$ & $g$ & $g$ & $f$ & $f$ & $g$ & $g$ \\
$c$ & $g$ & $g$ & $c$ & $c$ & $g$ & $g$ \\
$g$ & $g$ & $g$ & $g$ & $g$ & $g$ & $g$ \\
\hline
\end{tabular}

(1) Being $a^{2} * a * \operatorname{neut}\left(a^{2}\right)=a * \operatorname{neut}\left(a^{2}\right) * a^{2}=\operatorname{neut}\left(a^{2}\right), a^{2} * e * \operatorname{neut}\left(a^{2}\right)=e * \operatorname{neut}\left(a^{2}\right) * a^{2}=$ neut $\left(a^{2}\right)$, that is, for any $x \in\left\{\operatorname{anti}\left(a^{2}\right)\right\}, a^{2} * x *$ neut $\left(a^{2}\right)=x *$ neut $\left(a^{2}\right) * a^{2}=\operatorname{neut}\left(a^{2}\right)$.

(2) Being $b^{2} * \operatorname{neut}\left(b^{2}\right) * b=\operatorname{neut}\left(b^{2}\right) * b * b^{2}=\operatorname{neut}\left(b^{2}\right), b^{2} * \operatorname{neut}\left(b^{2}\right) * f=\operatorname{neut}\left(b^{2}\right) * f * b^{2}=$ neut $\left(b^{2}\right)$, that is, for any $x \in\left\{\operatorname{anti}\left(b^{2}\right)\right\}, b^{2} *$ neut $\left(b^{2}\right) * x=\operatorname{neut}\left(b^{2}\right) * x * b^{2}=\operatorname{neut}\left(b^{2}\right)$.

(3) Being neut $\left(\left(\right.\right.$ neut $\left.\left.\left(a^{2}\right)\right)\right)=\operatorname{neut}\left(a *\right.$ neut $\left.\left(a^{2}\right)\right)=\operatorname{neut}\left(a^{2}\right)$, neut $\left(\left(\operatorname{neut}\left(a^{2}\right)\right)\right)=\operatorname{neut}(e *$ $\left.\operatorname{neut}\left(a^{2}\right)\right)=\operatorname{neut}\left(a^{2}\right)$, that is, for any $x \in\left\{\operatorname{anti}\left(a^{n}\right)\right\} \cup\left\{a^{n}\right\}$, neut $\left(\left(\operatorname{neut}\left(a^{n}\right)\right)\right)=\operatorname{neut}(x *$ $\left.\operatorname{neut}\left(a^{n}\right)\right)=\operatorname{neut}\left(a^{n}\right)$.

(4) For each element $x, y, z \in S$, neut $\left(c^{2}\right) * x=y *$ neut $\left(c^{2}\right)=z *$ neut $\left(c^{2}\right)=g$, that is, for any $x, y, z \in\left\{\operatorname{anti}\left(c^{2}\right)\right\}, \operatorname{neut}\left(c^{2}\right) * x=y * \operatorname{neut}\left(c^{2}\right)=z *$ neut $\left(c^{2}\right)$.

(5) Being $b * f=b^{2}=f, f * f=b^{2}=f$, that is, for any $x \in\left\{\operatorname{anti}\left(b^{2}\right)\right\}$, anti $\left(x * \operatorname{neut}\left(b^{2}\right)\right) * \operatorname{neut}(x *$ $\left.\operatorname{neut}\left(b^{2}\right)\right)=b^{2}$. 
Proposition 9. Let $(N, *)$ be a commutative GNETG, then $\forall a, b \in N$, there are two positive integers $m$ andn such that the following hold:

(1) neut $\left(a^{n}\right) *$ neut $\left(b^{m}\right)=\operatorname{neut}\left(a^{n} * b^{m}\right)$.

(2) $\operatorname{anti}\left(a^{n}\right) * \operatorname{anti}\left(b^{m}\right) \in\left\{\operatorname{anti}\left(a^{n} * b^{m}\right)\right\}$.

Proof. Being $(N, *)$ be a commutative GNETG, then for $a \in N$, there is a positive integer $n$, such that $a^{n}$ exists the neutral element and opposite element, denoted by neut $\left(a^{n}\right)$ and anti $\left(a^{n}\right)$ respectively. For $b \in N$, there is a positive integer $m$, such that $b^{m}$ exists the and opposite element, denoted by neut $\left(b^{m}\right)$ and $\operatorname{anti}\left(b^{m}\right)$ respectively. So

$$
\begin{aligned}
\left(\operatorname{neut}\left(a^{n}\right) * \operatorname{neut}\left(b^{m}\right)\right) *\left(a^{n} * b^{m}\right) & =\left(\left(\operatorname{neut}\left(a^{n}\right) * \operatorname{neut}\left(b^{m}\right)\right) * a^{n}\right) * b^{m} \\
& =\left(\left(\operatorname{neut}\left(a^{n}\right) * a^{n}\right) * \operatorname{neut}\left(b^{m}\right)\right) * b^{m} \\
& =\left(a^{n} * \operatorname{neut}\left(b^{m}\right)\right) * b^{m} \\
& =a^{n} *\left(\operatorname{neut}\left(b^{m}\right) * b^{m}\right)=a^{n} * b^{m} .
\end{aligned}
$$

in the same way, we have $\left(a^{n} * b^{m}\right) *\left(\right.$ neut $\left(a^{n}\right) *$ neut $\left.\left(b^{m}\right)\right)=a^{n} * b^{m}$. That is:

$$
\left(a^{n} * b^{m}\right) *\left(\operatorname{neut}\left(a^{n}\right) * \operatorname{neut}\left(b^{m}\right)\right)=\left(\operatorname{neut}\left(a^{n}\right) * \operatorname{neut}\left(b^{m}\right)\right) *\left(a^{n} * b^{m}\right)=a^{n} * b^{m} .
$$

Moreover, for any $\operatorname{anti}\left(a^{n}\right) \in\left\{\operatorname{anti}\left(a^{n}\right)\right\}$ and $\operatorname{anti}\left(b^{m}\right) \in\left\{\operatorname{anti}\left(b^{m}\right)\right\}$, we can get,

$$
\begin{aligned}
\left(\operatorname{anti}\left(a^{n}\right) * \operatorname{anti}\left(b^{m}\right)\right) *\left(a^{n} * b^{m}\right) & =\left(\left(\operatorname{anti}\left(a^{n}\right) * \operatorname{anti}\left(b^{m}\right)\right) * a^{n}\right) * b^{m} \\
& =\left(\left(\operatorname{anti}\left(a^{n}\right) * a^{n}\right) * \operatorname{anti}\left(b^{m}\right)\right) * b^{m} \\
& =\left(\operatorname{neut}\left(a^{n}\right) * \operatorname{anti}\left(b^{m}\right)\right) * b^{m} \\
& =\operatorname{neut}\left(a^{n}\right) *\left(\operatorname{anti}\left(b^{m}\right) * b^{m}\right)=\operatorname{neut}\left(a^{n}\right) * \operatorname{neut}\left(b^{m}\right) .
\end{aligned}
$$

Similarly, we have $\left(a^{n} * b^{m}\right) *\left(\operatorname{anti}\left(a^{n}\right) * \operatorname{anti}\left(b^{m}\right)\right)=\operatorname{neut}\left(a^{n}\right) * n e u t\left(b^{m}\right)$. That is:

$$
\left(a^{n} * b^{m}\right) *\left(\operatorname{anti}\left(a^{n}\right) * \operatorname{anti}\left(b^{m}\right)\right)=\left(\operatorname{anti}\left(a^{n}\right) * \operatorname{anti}\left(b^{m}\right)\right) *\left(a^{n} * b^{m}\right)=\operatorname{neut}\left(a^{n}\right) * \operatorname{neut}\left(b^{m}\right) .
$$

Thus, we have

$$
\operatorname{neut}\left(a^{n}\right) * \operatorname{neut}\left(b^{m}\right) \in\left\{\text { neut }\left(a^{n} * b^{m}\right)\right\} .
$$

From this, by Proposition 7, we get: neut $\left(a^{n}\right) *$ neut $\left(b^{m}\right)=\operatorname{neut}\left(a^{n} * b^{m}\right)$. Therefore, we get anti $\left(a^{n}\right) *$ $\operatorname{anti}\left(b^{m}\right) \in\left\{\operatorname{anti}\left(a^{n} * b^{m}\right)\right\}$.

Example 8. Consider $\left(Z_{12}, \otimes\right)$, which is a commutative GNETG from Example 5. Without losing generality, select [2] and [4], then exist two positive integers 2 and 1 , such that neut $\left([2]^{2}\right) *$ neut $\left([4]^{1}\right)=$ neut $\left([2]^{2} *\right.$ $\left.[4]^{1}\right)=[4]$, so explain (1) of Proposition 9 is correct. Being $\{$ anti $[4]\}=\{[1],[4],[7]\}$ and $[1],[4],[7]$ is a sub algebra structure of $\left(Z_{12}, \otimes\right)$ by Table 5. So, explain (2) of Proposition 9 is correct.

Table 5. The operator table of $\{[1],[4],[7]\}$ in $\left(Z_{12}, \otimes\right)$.

\begin{tabular}{llll}
\hline$\otimes$ & {$[1]$} & {$[4]$} & {$[7]$} \\
\hline$[1]$ & {$[1]$} & {$[4]$} & {$[7]$} \\
{$[4]$} & {$[4]$} & {$[4]$} & {$[4]$} \\
{$[7]$} & {$[1]$} & {$[4]$} & {$[1]$} \\
\hline
\end{tabular}


Example 9. Apply the $(S, *)$ in Example 7 , Since it is not a commutative GNETG, we can get that:

(1) Being for each $2 \leq n \in Z^{+}, a^{n}=e$, and for each $m \in Z^{+}, f^{m}=f$, so neut $\left(a^{n}\right) * n e u t\left(f^{m}\right)=e * f=c$. On the other hand, neut $\left(a^{n} * f^{m}\right)=$ neut $(e * f)=$ neut $(c)$, but neut $(c)$ does not exist, so the (1) of Proposition 9 does not hold.

(2) Being $\left\{\operatorname{anti}\left(a^{n}\right)\right\}=\{a, e\},\left\{\operatorname{anti}\left(f^{m}\right)\right\}=\{f\}, a^{n} * f^{m}=c$, but anti(c) does not exist, we can get that the (2) of Proposition 9 does not hold.

Theorem 7. Let $(N, *)$ be a groupoid. Then $N$ is a GNETG if and only if it is a quasi-completely regular semigroup.

Proof. Necessity: Suppose $N$ is a GNETG, from Definition 10, for each $a \in N$, there is a positive integer $n$, such that $a^{n}$ exists the neutral element and opposite element, denoted by neut $\left(a^{n}\right)$ and $\operatorname{anti}\left(a^{n}\right)$ respectively. Set

$$
\left(a^{n}\right)^{-1}=\operatorname{anti}\left(a^{n}\right) * \operatorname{neut}\left(a^{n}\right),
$$

by Proposition $8,\left(a^{n}\right)^{-1}$ is unique and we have

$$
\begin{gathered}
\left(\left(a^{n}\right)^{-1}\right)^{-1}=\operatorname{anti}\left(\operatorname{anti}\left(a^{n}\right) * \operatorname{neut}\left(a^{n}\right)\right) * \operatorname{neut}\left(\operatorname{anti}\left(a^{n}\right) * \operatorname{neut}\left(a^{n}\right)\right)=a^{n}, \\
a^{n} *\left(a^{n}\right)^{-1} * a^{n}=a^{n} * \operatorname{anti}\left(a^{n}\right) * \operatorname{neut}\left(a^{n}\right) * a^{n}=a^{n}, \\
a^{n} * \operatorname{anti}\left(a^{n}\right) * \operatorname{neut}\left(a^{n}\right)=\operatorname{anti}\left(a^{n}\right) * \operatorname{neut}\left(a^{n}\right) * a^{n}=\operatorname{neut}\left(a^{n}\right), \text { i.e. } a^{n} *\left(a^{n}\right)^{-1}=\left(a^{n}\right)^{-1} * a^{n}
\end{gathered}
$$

from Definition $7, N$ is a quasi-completely regular semigroup.

Sufficiency: If $N$ is a completely quasi-regular semigroup. For any $a \in N$, there is a positive integer $n$ and $\left(a^{n}\right)^{-1} \in N$, such that $a^{n} *\left(a^{n}\right)^{-1} * a^{n}=a^{n}$ and $a^{n} *\left(a^{n}\right)^{-1}=\left(a^{n}\right)^{-1} * a^{n}$, set

$$
\operatorname{neut}\left(a^{n}\right)=a^{n} *\left(a^{n}\right)^{-1},
$$

then

$$
\begin{gathered}
\operatorname{neut}\left(a^{n}\right) * a^{n}=a^{n} *\left(a^{n}\right)^{-1} * a^{n}=a^{n}, \\
a^{n} * \operatorname{neut}\left(a^{n}\right)=a^{n} * a^{n} *\left(a^{n}\right)^{-1}=a^{n} *\left(a^{n}\right)^{-1} * a^{n}=a^{n}, \\
a^{n} *\left(a^{n}\right)^{-1}=\left(a^{n}\right)^{-1} * a^{n}=\operatorname{neut}\left(a^{n}\right) .
\end{gathered}
$$

From Definition 10, we have that $N$ is a GNETG and $\left(a^{n}\right)^{-1} \in\left\{\operatorname{anti}\left(a^{n}\right)\right\}$.

Example 10. Apply $(S, *)$ in the Example 7, we know that it is a GNETG. We will show that it is a quasi-completely regular semigroup in the following.

For $e$, there exists an inverse element $e^{-1}=e$, such that $\left(e^{-1}\right)^{-1}=e, e * e^{-1} * e=e, e * e^{-1}=e^{-1} * e$, so $e$ is completely regular. $f$ and $g$ are completely regular for the same reason. Moreover, being $a^{2}=e, b^{2}=f$ and $c^{2}=g$, so $a^{2}, b^{2}$ and $c^{2}$ are completely regular, so $(S, *)$ is a quasi-completely regular semigroup by Definition 7.

Definition 12. Let $(N, *)$ is a GNETG. $N$ is called a weak commutative GNETG if there are two positive integers $n, m$, such that $a^{n} *$ neut $\left(b^{m}\right)=n e u t\left(b^{m}\right) * a^{n}$ for all $a, b \in N$.

Example 11. Let $S=\{a, b, c, d, f\}$, an operation $*$ on $S$ is defined as following Table 6 . Since $(a, a, a),(b, a, b),(c, c, c)$ and $(f, f, f)$ are neutrosophic extended triplets, but $d$ does not exist the neutral element and opposite element. Thus, $S$ is not a NETG. Moreover, $d^{2}=c$ exists the neutral element and opposite element, so $(S, *)$ is GNETG and $(d, c, c)$ is a generalized neutrosophic extended triplet with degree 2 . We can infer that $(S, *)$ is a GNETG but not a NETG. Moreover, it is not a commutative GNETG being $b * d \neq d * b$, we can show that it is a weak commutative GNETG. 
For $a, b, c, d$, and $f$, there exist positive integers $1,1,1,2$, and 1 respectively, so $S^{\prime}=\left\{a^{1}, b^{1}, c^{1}, d^{2}, f^{1}\right\}=$ $\{a, b, c, f\}$ being $d^{2}=c$. We know that neut $(a)=a, \operatorname{neut}(b)=a$,neut $(c)=c$,neut $(f)=f$, so $\{$ neut $(a)$, neut $(b)$, neut $(c)$, neut $(f)\} \in S^{\prime}$. From Table 6 , we can get the sub algebra system $\left(S^{\prime}, *\right)$ of $(S, *)$ as following Table 7 , and $\left(S^{\prime}, *\right)$ is commutative. Thus, $(S, *)$ is a weak commutative GNETG.

Table 6. The operation table of Example 11.

\begin{tabular}{llllll}
\hline$*$ & $a$ & $b$ & $c$ & $d$ & $f$ \\
\hline$a$ & $a$ & $b$ & $c$ & $d$ & $f$ \\
$b$ & $b$ & $a$ & $c$ & $d$ & $f$ \\
$c$ & $c$ & $c$ & $c$ & $c$ & $c$ \\
$d$ & $c$ & $c$ & $c$ & $c$ & $c$ \\
$f$ & $f$ & $f$ & $c$ & $d$ & $f$ \\
\hline
\end{tabular}

Table 7. The sub algebra system $S^{\prime}$ of $S$ in Example 11.

\begin{tabular}{lllll}
\hline$*$ & $a$ & $b$ & $c$ & $f$ \\
\hline$a$ & $a$ & $b$ & $c$ & $f$ \\
$b$ & $b$ & $a$ & $c$ & $f$ \\
$c$ & $c$ & $c$ & $c$ & $c$ \\
$f$ & $f$ & $f$ & $c$ & $f$ \\
\hline
\end{tabular}

Example 12. Select the $(S, *)$ in Example 4 , being $e^{n}=e, f^{m}=f$ for every $n, m \in Z^{+}, e^{n} *$ neut $\left(f^{m}\right)=$ $e * f=e, f^{m} * \operatorname{neut}\left(e^{n}\right)=f * e=f$, so $e^{n} * \operatorname{neut}\left(f^{m}\right)=e \neq f=\operatorname{neut}\left(f^{m}\right) * e^{n}, \forall n, m \in Z^{+}$. Thus, the $(S, *)$ in Example 4 is not weak commutative GNETG.

Proposition 10. Let $(N, *)$ be a GNETG. Then $(N, *)$ is a weak commutative GNETG iff there are two positive integers $n$ and $m$, such that $N$ satisfies the following conditions:

(1) neut $\left(a^{n}\right) * \operatorname{neut}\left(b^{m}\right)=\operatorname{neut}\left(b^{m}\right) * \operatorname{neut}\left(a^{n}\right)$, for all $a, b \in N$.

(2) neut $\left(a^{n}\right) * \operatorname{neut}\left(b^{m}\right) * a^{n}=a^{n} *$ neut $\left(b^{m}\right)$, for all $a, b \in N$.

Proof. Necessity: If $(N, *)$ is a weak commutative GNETG, then there are two positive integers $n, m$, such that $a^{n}$ and $b^{m}$ exist the neutral element and opposite element. So, from Proposition 7 and Definition 12, we have

$$
\operatorname{neut}\left(a^{n}\right) * \operatorname{neut}\left(b^{m}\right)=\operatorname{neut}\left(b^{m}\right) * \operatorname{neut}\left(a^{n}\right), \forall a, b \in N .
$$

Moreover,

$$
\begin{aligned}
\operatorname{neut}\left(a^{n}\right) * \operatorname{neut}\left(b^{m}\right) * a^{n} & =\operatorname{neut}\left(a^{n}\right) *\left(\operatorname{neut}\left(b^{m}\right) * a^{n}\right) \\
& =\operatorname{neut}\left(a^{n}\right) *\left(a^{n} * \operatorname{neut}\left(b^{m}\right)\right) \\
& =\left(\operatorname{neut}\left(a^{n}\right) * a^{n}\right) * \operatorname{neut}\left(b^{m}\right)=a^{n} * \operatorname{neut}\left(b^{m}\right)
\end{aligned}
$$

Sufficiency: Suppose that $N$ satisfies the conditions (1) and (2) above. Then:

$$
\begin{aligned}
a^{n} * \operatorname{neut}\left(b^{m}\right) & =\left(\operatorname{neut}\left(a^{n}\right) * \operatorname{neut}\left(b^{m}\right)\right) * a^{n} \\
& =\left(\operatorname{neut}\left(b^{m}\right) * \operatorname{neut}\left(a^{n}\right)\right) * a^{n} \\
& =\operatorname{neut}\left(b^{m}\right) *\left(\operatorname{neut}\left(a^{n}\right) * a^{n}\right)=\operatorname{neut}\left(b^{m}\right) * a^{n} .
\end{aligned}
$$

From Definition 12 we know that $(N, *)$ is a weak commutative GNETG. 
Proposition 11. Let $(N, *)$ be a weak commutative GNETG, then for $a, b \in N$, the following conditions are satisfied:

(1) neut $\left(a^{n}\right) *$ neut $\left(b^{m}\right)=\operatorname{neut}\left(b^{m} * a^{n}\right)$;

(2) $\operatorname{anti}\left(a^{n}\right) * \operatorname{anti}\left(b^{m}\right) \in\left\{\operatorname{anti}\left(b^{m} * a^{n}\right)\right\}$.

Proof. If $a, b \in N$, then there are two positive integers $m, n$, such that $a^{n}$ and $b^{m}$ exist the neutral element and opposite element. We have

$$
\begin{aligned}
\left(\operatorname{neut}\left(a^{n}\right) * \operatorname{neut}\left(b^{m}\right)\right) *\left(b^{m} * a^{n}\right) & =\left(\left(\operatorname{neut}\left(a^{n}\right) * \operatorname{neut}\left(b^{m}\right)\right) * b^{m}\right) * a^{n} \\
& =\left(\operatorname{neut}\left(a^{n}\right) *\left(b^{m} * \operatorname{neut}\left(b^{m}\right)\right)\right) * a^{n} \\
& =\left(\operatorname{neut}\left(a^{n}\right) * b^{m}\right) * a^{n} \\
& =\left(b^{m} * \operatorname{neut}\left(a^{n}\right)\right) * a^{n}=b^{m} *\left(\operatorname{neut}\left(a^{n}\right) * a^{n}\right)=b^{m} * a^{n} .
\end{aligned}
$$

Similarly, we have $\left(b^{m} * a^{n}\right) *\left(\right.$ neut $\left(a^{n}\right) *$ neut $\left.\left(b^{m}\right)\right)=\left(\right.$ neut $\left(a^{n}\right) *$ neut $\left.\left(b^{m}\right)\right) *\left(b^{m} * a^{n}\right)=b^{m} * a^{n}$. Moreover, for any $\operatorname{anti}\left(a^{n}\right) \in\left\{\operatorname{anti}\left(a^{n}\right)\right\}$ and $\operatorname{anti}\left(b^{m}\right) \in\left\{\operatorname{anti}\left(b^{m}\right)\right\}$, we have:

$$
\begin{aligned}
\left(\operatorname{anti}\left(a^{n}\right) * \operatorname{anti}\left(b^{m}\right)\right) *\left(b^{m} * a^{n}\right) & =\left(\left(\operatorname{anti}\left(a^{n}\right) * \operatorname{anti}\left(b^{m}\right)\right) * b^{m}\right) * a^{n} \\
& =\left(\operatorname{anti}\left(a^{n}\right) *\left(\operatorname{anti}\left(b^{m}\right) * b^{m}\right)\right) * a^{n} \\
& =\left(\operatorname{anti}\left(a^{n}\right) * \operatorname{neut}\left(b^{m}\right)\right) * a^{n} \\
& =\operatorname{anti}\left(a^{n}\right) *\left(\operatorname{neut}\left(b^{m}\right) * a^{n}\right) \\
& =\operatorname{anti}\left(a^{n}\right) *\left(a^{n} * \operatorname{neut}\left(b^{m}\right)\right) \\
& =\left(\operatorname{anti}\left(a^{n}\right) * a^{n}\right) * \operatorname{neut}\left(b^{m}\right)=\operatorname{neut}\left(a^{n}\right) * \operatorname{neut}\left(b^{m}\right) .
\end{aligned}
$$

Similarly, we have $\left(b^{m} * a^{n}\right) *\left(\operatorname{anti}\left(a^{n}\right) * \operatorname{anti}\left(b^{m}\right)\right)=\left(\operatorname{anti}\left(a^{n}\right) * \operatorname{anti}\left(b^{m}\right)\right) *\left(b^{m} * a^{n}\right)=$ neut $\left(a^{n}\right) *$ neut $\left(b^{m}\right)$. So, we have neut $\left(a^{n}\right) *$ neut $\left(b^{m}\right) \in\left\{\right.$ neut $\left.\left(b^{m} * a^{n}\right)\right\}$. From this, we can get $\operatorname{neut}\left(a^{n}\right) * \operatorname{neut}\left(b^{m}\right)=\operatorname{neut}\left(b^{m} * a^{n}\right)$. Thus, we have anti $\left(a^{n}\right) * \operatorname{anti}\left(b^{m}\right) \in \operatorname{anti}\left(b^{m} * a^{n}\right)$.

Example 13. Let $S=\{a, e, b, f, c, g\}$, an operation $*$ on $S$ is defined as following Table 8. Being netu $(e)=$ $e,\{\operatorname{anti}(e)\}=\{a, e\}, \operatorname{netu}(f)=f,\{\operatorname{anti}(f)\}=\{b, f\}, \operatorname{netu}(g)=g,\{\operatorname{anti}(g)\}=\{a, e, b, f, c, g\}$ and $a^{2}=e, b^{2}=f, c^{2}=g$, so $(S, *)$ is a GNETG. It is easy to verify $(S, *)$ is a weak commutative GNETG by Definition 12. We also can get:

(1) Being neut $\left(b^{2}\right) * \operatorname{neut}\left(e^{1}\right)=f * e=g$, and neut $\left(e^{1} * b^{2}\right)=\operatorname{neut}(g)=g$, so, neut $\left(b^{2}\right) * \operatorname{neut}\left(e^{1}\right)=$ $\operatorname{neut}\left(e^{1} * b^{2}\right)$.

(2) Being $\left\{\operatorname{anti}\left(b^{2}\right)\right\}=\{b, f\},\left\{\operatorname{anti}\left(e^{1}\right)\right\}=\{a, e\},\left\{\operatorname{anti}\left(b^{2} * e^{1}\right)\right\}=\{\operatorname{anti}(g)\}=\{a, e, b, f, c, g\}$, thus, anti $\left(b^{2}\right) * \operatorname{anti}\left(e^{1}\right) \in\left\{\operatorname{anti}\left(b^{2} * e^{1}\right)\right\}$.

Table 8. The operation table of Example 13.

\begin{tabular}{lllllll}
\hline$*$ & $a$ & $e$ & $b$ & $f$ & $c$ & $g$ \\
\hline$a$ & $e$ & $e$ & $c$ & $c$ & $g$ & $g$ \\
$e$ & $e$ & $e$ & $g$ & $g$ & $g$ & $g$ \\
$b$ & $c$ & $g$ & $f$ & $f$ & $c$ & $g$ \\
$f$ & $c$ & $g$ & $f$ & $f$ & $c$ & $g$ \\
$c$ & $g$ & $g$ & $c$ & $c$ & $g$ & $g$ \\
$g$ & $g$ & $g$ & $g$ & $g$ & $g$ & $g$ \\
\hline
\end{tabular}

Example 14. Apply the $(S, *)$ in Example 4, Being it is not a weak commutative GNETG, we can get that:

(1) Being neut $\left(a^{2}\right) * \operatorname{neut}\left(f^{1}\right)=e * f=e$, but neut $\left(f^{1} * a^{2}\right)=\operatorname{neut}(f)=f$, so, neut $\left(a^{2}\right) * \operatorname{neut}\left(f^{1}\right) \neq$ $\operatorname{neut}\left(f^{1} * a^{2}\right)$. 
(2) Being $\left\{\operatorname{anti}\left(a^{2}\right)\right\}=\{a, e\},\left\{\operatorname{anti}\left(f^{1}\right)\right\}=\{f\},\left\{\operatorname{anti}\left(f^{1} * a^{2}\right)\right\}=\{\operatorname{anti}(f)\}=\{f\}$, thus, $\operatorname{anti}\left(a^{2}\right) *$ $\operatorname{anti}\left(f^{1}\right) \notin\left\{\operatorname{anti}\left(f^{1} * a^{2}\right)\right\}$.

Definition 13. A semigroup $S$ is called a quasi-Clifford semigroup, if it is quasi-completely regular and for any $a, b \in S$, there are two positive integers $n, m$ such that

$$
a^{n} *\left(b^{m} *\left(b^{m}\right)^{-1}\right)=\left(b^{m} *\left(b^{m}\right)^{-1}\right) * a^{n} .
$$

Theorem 8. Let $(N, *)$ be a groupoid. Then $N$ is a weak commutative GNETG iff it is a quasi-Clifford semigroup.

Proof. Necessity: Suppose that $N$ is a weak commutative GNETG. By Theorem 7, we know that $N$ is a quasi-completely regular semigroup, then there are two positive integers $m, n$, such that $a^{n}$ and $b^{m}$ exist the neutral element and opposite element. Set

$$
\left(a^{n}\right)^{-1}=\operatorname{anti}\left(a^{n}\right) * \operatorname{neut}\left(a^{n}\right) .
$$

For any $a, b \in N$, so we have

$$
a^{n} *\left(b^{m} *\left(b^{m}\right)^{-1}\right)=\left(b^{m} *\left(b^{m}\right)^{-1}\right) * a^{n} .
$$

From Definition 13, we know that $N$ is a quasi-Clifford semigroup.

Sufficiency: Assume that $N$ is a quasi-Clifford semigroup, then there are two positive integers $m$ and $n$, such that $a^{n}$ and $b^{m}$ are completely regular. Then there exists $\left(a^{n}\right)^{-1}$ and $\left(b^{m}\right)^{-1}$. Set

$$
\operatorname{neut}\left(a^{n}\right)=a^{n} *\left(a^{n}\right)^{-1}, \operatorname{neut}\left(b^{m}\right)=b^{m} *\left(b^{m}\right)^{-1} .
$$

Applying Definition 13, being $a^{n} *\left(b^{m} *\left(b^{m}\right)^{-1}\right)=\left(b^{m} *\left(b^{m}\right)^{-1}\right) * a^{n}$, we have $a^{n} *$ neut $\left(b^{m}\right)=$ neut $\left(b^{m}\right) * a^{n}$, we can get that $N$ is a weak commutative GNETG by Definition 12 .

Example 15. Apply the $(S, *)$ in Example 11, Being it is a weak commutative GNETG from Example 11. We show that it is a quasi-Clifford semigroup. From Theorem 7 , we can see that $(S, *)$ is a quasi-completely regular semigroup, we just show for any $x, y \in S$, there are two positive integers $n$ and $m$ such that $x^{n} *\left(y^{m} *\right.$ $\left.\left(y^{m}\right)^{-1}\right)=\left(y^{m} *\left(y^{m}\right)^{-1}\right) * x^{n}$.

From Example 11, for $a, b, c, d$, and $f$, there exist positive integers $1,1,1,2$, and 1 respectively, and set $a^{-1}=a, b^{-1}=b, c^{-1}=c,\left(d^{2}\right)^{-1}=c, f^{-1}=f$. For any $x, y \in\left\{a^{1}, b^{1}, c^{1}, d^{2}, f^{1}\right\}$, without losing generality, let $x=a, y=b$, we can get $a^{1} *\left(b^{1} *\left(b^{1}\right)^{-1}\right)=\left(b^{1} *\left(b^{1}\right)^{-1}\right) * a^{1}=b$. We can verify other cases, thus $(S, *)$ is a quasi-Clifford semigroup.

Example 16. Apply the $(S, *)$ in Example 4, Being it is not a weak commutative GNETG from Example 12. We show that there exists $x, y \in S$, for any two positive integers $n$ and $m$ such that $x^{n} *\left(y^{m} *\left(y^{m}\right)^{-1}\right) \neq$ $\left(y^{m} *\left(y^{m}\right)^{-1}\right) * x^{n}$.

From Example 4, for any $n, m \in Z^{+}, e^{n}=e, f^{m}=f$ and $\left(e^{n}\right)^{-1}=e,\left(f^{m}\right)^{-1}=f$, but $e^{n} *\left(f^{m} *\right.$ $\left.\left(f^{m}\right)^{-1}\right)=e \neq f=\left(f^{m} *\left(f^{m}\right)^{-1}\right) * e^{n}$. That is for $e, f \in S$, there are not two positive integers $n, m$ such that $e^{n} *\left(f^{m} *\left(f^{m}\right)^{-1}\right)=\left(f^{m} *\left(f^{m}\right)^{-1}\right) * e^{n}$. So $(S, *)$ is not a quasi-Clifford semigroup.

\section{Conclusions}

In the paper, from the perspective of semigroup theory, we deeply studied the GNETG and obtained some important results. We proved that the GNETG is equal to the quasi-completely regular semigroup, and the weak commutative GNETG is equal to the quasi-Clifford semigroup. Moreover, we investigated the relationship between $\left(Z_{n}, \otimes\right)$ and a NETG. All these results are interesting for exploring the structure characterization of GNETG. As the next research topics, we will explore the 
structure of some special GNETG and their relationships with related logic algebras. Moreover, we will discuss the integration of the related topics, such as the combination of neutrosophic set, fuzzy set, soft set and algebra systems [23,24].

Author Contributions: All authors have contributed equally to this paper.

Funding: This research was funded by National Natural Science Foundation of China (Grant No. 11501435), Instructional Science and Technology Plan Projects of China National Textile and Apparel Council (No. 2016073) and Scientific Research Program Funded by Shaanxi Provincial Education Department (Program No. 18JS042).

Acknowledgments: The authors would like to thank the reviewers for their many insightful comments and suggestions.

Conflicts of Interest: The authors declare no conflicts of interest.

\section{References}

1. Smarandache, F.; Ali, M. Neutrosophic triplet group. Neural Comput. Appl. 2018, 29, 595-601. [CrossRef]

2. Vasantha, W.B.; Kandasamy, I.; Smarandache, F. Neutrosophic Triplet Groups and Their Applications to Mathematical Modelling; EuropaNova: Brussels, Belgium, 2017; ISBN 978-1-59973-533-7.

3. Smarandache, F. Hybrid Neutrosophic Triplet Ring in Physical Structures. Bull. Am. Phys. Soc. 2017, 62, 17.

4. Bal, M.; Shalla, M.M.; Olgun, N. Neutrosophic Triplet Cosets and Quotient Groups. Symmetry 2017, 10, 126. [CrossRef]

5. Smarandache, F.; Ali, M. Neutrosophic Triplet Ring and its Applications. In Proceedings of the 18th Annual Meeting of the APS Northwest Section, Pacific University, Forest Grove, OR, USA, 1-3 June 2017.

6. Sahin, M.; Abdullah, K. Neutrosophic triplet normed space. Open Phys. 2017, 15, 697-704. [CrossRef]

7. Jaiyeola, T.G.; Smarandache, F. Some results on neutrosophic triplet group and their applications. Symmetry 2018, 10, 202. [CrossRef]

8. Şahin, M.; Kargin, A. neutrosophic triplet v-generalized metric space. Axioms 2018, 7, 67. [CrossRef]

9. Ali, M.; Smarandache, F.; Khan, M. Study on the development of neutrosophic triplet ring and neutrosophic triplet field. Mathematics 2018, 6, 46. [CrossRef]

10. Gulistan, M.; Nawaz, S.; Hassan, N. Neutrosophic triplet non-associative semihypergroups with application. Symmetry 2018, 10, 613. [CrossRef]

11. Smarandache, F. Neutrosophic Perspectives: Triplets, Duplets, Multisets, Hybrid Operators, Modal Logic, Hedge Algebras. And Applications; Pons Publishing House: Brussels, Belgium, 2017.

12. Zhang, X.H.; Bo, C.X.; Smarandache, F.; Dai, J.H. New inclusion relation of neutrosophic sets with applications and related lattice structure. Int. J. Mach. Learn. Cybern. 2018, 9, 1753-1763. [CrossRef]

13. Zhang, X.H.; Bo, C.X.; Smarandache, F.; Park, C. New operations of totally dependent-neutrosophic sets and totally dependent-neutrosophic soft sets. Symmetry 2018, 10, 187. [CrossRef]

14. Zhang, X.H.; Wu, X.Y.; Smarandache, F.; Hu, M.H. Left (right)-quasi neutrosophic triplet loops (groups) and generalized BE-algebras. Symmetry 2018, 10, 241. [CrossRef]

15. Zhang, X.; Hu, Q.; Smarandache, F; An, X. On Neutrosophic Triplet Groups: Basic Properties, NT-Subgroups, and Some Notes. Symmetry 2018, 10, 289. [CrossRef]

16. Zhang, X.H.; Smarandache, F.; Liang, X.L. Neutrosophic Duplet Semi-Group and Cancellable Neutrosophic Triplet Groups. Symmetry 2017, 9, 275. [CrossRef]

17. Zhang, X.H.; Wang, X.J.; Smarandache, F.; Jaíyéolá, T.G.; Liang, X.L. Singular neutrosophic extended triplet groups and generalized groups. Cognit. Syst. Res. 2018, in press. [CrossRef]

18. Zhang, X.H.; Wu, X.Y.; Mao, X.Y.; Smarandache, F.; Park, C. On Neutrosophic Extended Triplet Groups (Loops) and Abel-Grassmann's Groupoids (AG-Groupoids). J. Intell. Fuzzy Syst. 2019, in press.

19. Vasantha, W.B.; Kandasamy, I.; Smarandache, F. A Classical Group of Neutrosophic Triplet Groups Using $\left\{Z_{2 p}, \times\right\}$. Symmetry 2018, 10, 193.

20. Vasantha, W.B.; Kandasamy, I.; Smarandache, F. Neutrosophic Duplets of $\left\{Z_{p^{n}}, \times\right\}$ and $\left\{Z_{p q}, \times\right\}$ and Their Properties. Symmetry 2018, 10, 344.

21. Akinmoyewa, J.T. A study of some properties of generalized groups. Octogon Math. Mag. 2009, 17, 599-626.

22. Howie, J.M. Fundamentals of Semigroup Theory; Oxford University Press: Oxford, UK, 1995. 
23. Zhang, X.H. Fuzzy anti-grouped filters and fuzzy normal filters in pseudo-BCI algebras. J. Intell. Fuzzy Syst. 2017, 33, 1767-1774. [CrossRef]

24. Zhang, X.H.; Park, C.; Wu, S.P. Soft set theoretical approach to pseudo-BCI algebras. J. Intell. Fuzzy Syst. 2018, 34, 559-568. [CrossRef]

(C) 2019 by the authors. Licensee MDPI, Basel, Switzerland. This article is an open access article distributed under the terms and conditions of the Creative Commons Attribution (CC BY) license (http:/ / creativecommons.org/licenses/by/4.0/). 